\title{
SENSOR DE PISCADAS PARA COMUNICAÇÃO ENTRE PACIENTE E CUIDADOR.
}

\author{
João Paulo Silveira dos Santos* e Euler Vilhena de Garcia* \\ *Departamento de Pós-Graduação em Engenharia Biomédica/Universidade de Brasília, Brasília, \\ Brasil \\ e-mail: joao123@gmail.com
}

\begin{abstract}
Resumo: Este trabalho tem como objetivo verificar a possibilidade de interpretar o ato de piscar os olhos para criar um meio de comunicação entre paciente e cuidador. Um dispositivo de controle foi projetado e desenvolvido utilizando um sensor de infravermelho e um LED infravermelho apontados para o olho do paciente instalados na armação de um óculos. A interpretação da informação é feita através de um dispositivo similar ao Arduino nano e a informação é apresentada em um display de LCD. Ao ocorrer um piscada o dispositivo altera a informação apresentada no display, facilitando a troca de informações básicas entre paciente e cuidador. Como o led infravermelho é um emissor de calor, foi observado que a potência emitida pelo sistema está em níveis seguros sendo muito inferior aos valores máximos estipulados em norma. Os testes mostraram que o sistema é viável sendo possível o desenvolvimento e aperfeiçoamento de um dispositivo de interpretação de piscadas com o uso de infravermelho.
\end{abstract}

Palavras-chave: Arduino, piscar de olhos, sensor de infravermelho, LED infravermelho.

\begin{abstract}
This paper aims to verify the possibility of eye blinking interpretation to create a means of communication between patient and caregiver. $A$ control device was designed and developed using an infrared sensor and an infrared LED pointed towards the patient's eye installed in an eyeglass frame. The interpretation information is made by Arduino nano similar device and information is presented on an LCD display. When there is a blink the device changes the information displayed on the display, facilitating the exchange of basic informations between patient and caregiver. As the infrared LED is a heat emitter, it was observed that the power emitted by the system is at safe levels being much lower than the maximum values stipulated in the standards. The tests showed that the development of the device using infrared for blink interpretation is possible.
\end{abstract}

Keywords: Arduino, eye blinking, infrared sensor, infrared $L E D$.

\section{Introdução}

$\mathrm{O}$ ato de piscar os olhos tem função de renovar as lágrimas, proteger os olhos do contato com objetos externos e etc.

Fechamentos e reaberturas rápidas ocorrem espontaneamente sem necessariamente existir um estimulo externo.

O Piscar de olhos espontâneo é um indicador acessível para fadiga. Possui diversas vantagens uma vez que representa um ato normal simples de ser observado, sendo um fenômeno que reflete a influência do centro ativação nervosa sem manipulação voluntária[1].

Estudos recentes demonstram que a análise de piscadas espontâneas pode fornecer informação substancial sobre os processos de ativação do sistema nervoso central e fadiga[1]. Os parâmetros frequência, de intermitência e duração das piscadas são especialmente sujeitos a modificações características com o aumento sedação ou sonolência.

Doenças como a esclerose lateral amiotrófica (ELA) afetam o sistema nervoso, de forma degenerativa e incapacitante, caracterizada por perda de neurônios motores no córtex, tronco cerebral, e medula espinhal[2].

Pacientes portadores de ELA em estado avançado, tem perda total dos movimentos, perda da fala e etc, o que dificulta o cuidado já que esses pacientes precisam de atenção e cuidados constantes. Os pacientes que conservam seu intelecto e lucidez, utilizam o olhar, o piscar ou expressões faciais como elementos essenciais de comunicação[2].

O dispositivo criado visa desenvolver um meio simples de comunicação entre paciente e seu cuidador com o uso de piscadas, tornando precisa e fácil a troca de informações.

\section{Materiais e métodos}

O dispositivo criado tem o intuito de transmitir uma informação do que o paciente está sentindo para seu cuidador. Através de um contador de piscadas é possível que o paciente transmita/altere a mensagem, préprogramada no dispositivo. 
Foram criadas inicialmente 6 sensações básicas: Felicidade, Dor, Fome, Sede, Calor e Frio, que são apresentados no display.

O protótipo criado consiste em um fototransistor de infravermelho iluminado por um led infravermelho. O conjunto sensor ótico reflexível com fototransistor de saída utilizado foi o da fabricante VISHAY modelo TCRT5000 fixado aos óculos de proteção individual, conforme ilustra a figura 1 .



Figura 1: Modo de fixação do sensor aos óculos.

Para exibir a informação desejada foi utilizado um display LCD 16x2. O processamento da informação é feito por um dispositivo similar ao Arduino Nano baseado no microcontrolador ATmega328 da fabricante ATMEL.

As piscadas são identificadas através da variação do valor lido da saída do fototransistor, que está ligado a uma entrada analógica do Arduino. Ao ocorrer uma piscada o movimento do olho faz com que a quantidade de luz infravermelha refletida pelo olho e absorvida pelo fototransistor mude, o resultado é que sua saída de tensão terá seu valor alterado. O Arduino recebe em sua entrada analógica uma variação de tensão, ao ser detectado um flanco de subida o sistema interpreta como uma piscada.

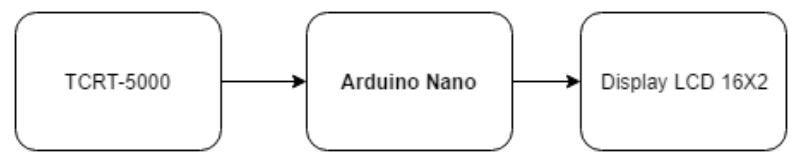

Figura 2: Diagrama de blocos do sistema

O contador de piscadas analisa a quantidade de piscadas e altera a sensação que é apresentada no display de lcd, a atualização da sensação apresentada é feita de forma incremental, onde cada vez que o paciente piscar a sensação é atualizada. A figura 3 ilustra como são apresentadas a informações no display LCD.

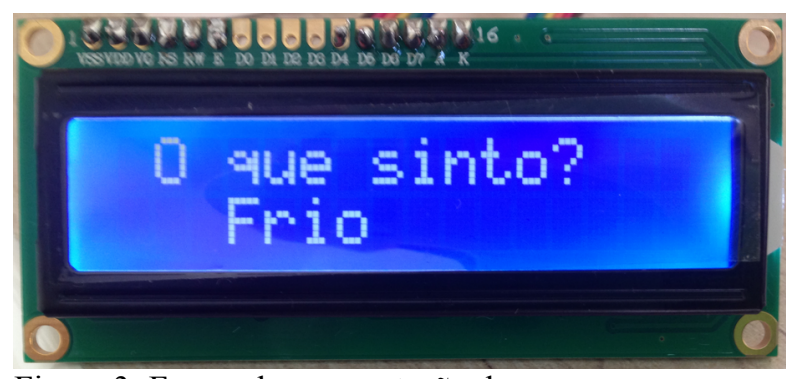

Figura 3: Forma de apresentação da mensagem.

\section{Resultados}

O protótipo mostrou-se sensível as piscadas, sendo apresentada uma nova informação cada vez que o paciente piscar.

Toda vez que o paciente quiser apresentar um sentimento é necessário que ele pisque por vezes seguidas até chegar a sensação desejada.

O teste do dispositivo foi realizado por duas pessoas, cada vez que ocorre uma piscada era atualizada a mensagem no display LCD.

\section{Discussão}

Os testes realizados mostraram que o dispositivo ainda está muito sensível a variação da reflexão de luz emitida pelo led infravermelho, necessitando de um ajuste fino. No entanto o protótipo mostrou a viabilidade da ideia e validade do projeto.

Durante os testes foi possível perceber que o dispositivo respondia as piscadas voluntárias, quando a pessoa desejava alterar mensagem, mas também alterava a mensagem quando ocorriam piscadas espontâneas, ou seja, quando a pessoa não desejava alterar a mensagem, isso ocorre, pois, o dispositivo inicialmente detecta apenas a variação da intensidade sem verificar o tempo de duração da piscada.

Deve-se observar que o led infravermelho é um emissor de calor. Devendo-se respeitar os níveis de exposição propostos pela da IEC- 62471, Segurança fotobiológica de lâmpadas e sistemas de lâmpadas, cláusula 4.3. As especificações limite de exposição relevantes para componentes com emissão próxima de infravermelho são fornecidos na cláusula 4.3.5, limite de exposição térmica perigosa a retina.

Como o uso do equipamento é proposto para um tempo maior que 1000s um valor limite de exposição da córnea ao infravermelho normatizado pela IEC-62471 fixado é menor que $100 \mathrm{~W} / \mathrm{m}^{2}$ [3].

Segundo os dados fornecidos pelo datasheet do sensor ótico reflexivo com transistor de saída TCRT5000, a intensidade irradiada pelo led infravermelho é de $\mathrm{Ie}=0,021 \mathrm{~W} / \mathrm{sr}$ com comprimento de onda $\lambda=940 \mathrm{~nm}$ [4]. A distância do led infravermelho ao olho do paciente é de $0,1 \mathrm{~m}$.

A irradiância pode ser calculada a partir da intensidade de radiação Ie e distância d a partir da seguinte equação: 


$$
E_{e}=\frac{I_{e}}{d^{2}}=\frac{0,021}{0,05^{2}}=8,4 \frac{\mathrm{W}}{\mathrm{m}^{2}}
$$

O resultado da equação 1 demonstra que a potência em que o olho do paciente está exposto é bem inferior aos $100 \mathrm{~W} / \mathrm{m}^{2}$ que recomenda a norma IEC- 62471 .

Dessa forma a emissão de luz infravermelho direta e continuamente pelo dispositivo está em nível de segurança aceitável, não gerando riscos a saúde do paciente.

\section{Conclusão}

$\mathrm{O}$ projeto proposto mostrou ser possível o desenvolvimento de um dispositivo para facilitar a comunicação entre cuidador e paciente nos casos em que o paciente tem limitações de fala e de movimentos, como é o caso de paciente em estágio avançado de esclerose lateral amiotrófica (ELA). Para confirmar a viabilidade será necessário um aperfeiçoamento do dispositivo e o teste em voluntários.

O protótipo necessita de aperfeiçoamento de modo que se crie uma sequência de piscadas para que se chegue a informação desejada, por exemplo, quando o paciente desejar apresentar uma informação ele piscar por duas vezes de forma lenta. Após com 1 piscada seja apresentada uma informação, com duas piscadas outra informação e assim por diante. Dessa forma, será possível uma comunicação mais precisa entre paciente e cuidador.

Para projetos futuros iremos aperfeiçoar o dispositivo para o acompanhamento do tempo das piscadas e estudo da variação da sonolência, fadiga e sedação de usuários. De forma que seja feito o acompanhamento parâmetros frequência, de intermitência e duração das piscadas.

\section{Agradecimentos}

Agradecemos ao departamento de Pós-Graduação em Engenharia Biomédica/UnB.

\section{Referências}

[1] Caffier, P. P., Erdmann, U. \& Ullsperger, P. Experimental evaluation of eye-blink parameters as a drowsiness measure. European journal of applied physiology 89, 319-325 (2003).

[2]Cortez, João Mario. "Vivendo com E.L.A.”, 2006

Disponível em:<

http://joaomario.blogspot.com.br/2006/03/comunica o-visual.html > Acesso em 07/07/2016

[3] Intersil TM. Eye Safety for Proximity Sensing Using Infrared Light-emitting Diodes. Application Note 1737, 2015

[4] Vishay Semiconductors Datasheet: TCRT5000, Reflective Optical Sensor with Transistor Output. Datasheet 